\title{
Etude anatomique et histologique (Cestodes) \\ de trois Raillietina s. str. d'Oiseaux africains
}

\author{
Par Jean-Claude QUENTIN
}

Jusqu'à présent, seules cinq espèces de Cestodes du genre Raillietina appartenant au sous-genre Raillietina ont été décrites chez les Cuculiformes.

Nous avons observé récemment l'une d'entre elles et de plus une espèce que nous considérons comme nouvelle, provenant de l'autopsie d'un Touraco (Cuculiforme). Elles nous sont envoyées de Côte-d'Ivoire par le Docteur J.-P. Doucet que nous remercions vivement.

Nous avons jugé intéressant de joindre à ces deux descriptions celle d'un Raillietina du même sous-genre, parasite d'un Vinago waahli (Columbiforme), originaire d'Afrique, en provenance du Zoo de Milan.

\section{RAILLIETINA (RAILLIETINA) KHALILI HILMY 1936}

MATÉRIEl ÉTUdí́: une dizaine d'individus rarement entiers, mais possédant tous leur scolex, plus des fragments de strobiles comportant des proglottis gravides, récoltés chez Turacus sp. (Cuculiforme), J.-P. Doucet, leg., 22 janvier 1955, Côte-d'Ivoire.

\section{1) DESCRIPTION :}

Nos exemplaires sont minces, longs de 12 à $13 \mathrm{~cm}$ environ lorsqu'ils ne sont pas contractés ; ils présentent une largeur maximale relevée au niveau des anneaux gravides de 1.000 à $1.100 \mu$. Le nombre total des proglottis a pu être dénombré sur deux échantillons. Il s'élève à 900 (884-956).

1. Scolex (fig. 1).

Nettement séparé du strobile, d'une largeur moyenne de 480-500 $\mu$ au niveau des ventouses, il atteint une hauteur de $350 \mu$. Le rostellum est resté invaginé sur les différents scolex que nous avons étudiés. En vue apicale, la double couronne de crochets, bien étalée entre lame et lamelle, présente un diamètre externe de 250-260 $\mu$, un diamètre interne de 190-200 $\mu$. Le nombre total de crochets peut être évalué entre 280 et 300. Sur quatre scolex, nous avons compté successivement 280, 291, 295, et 297 crochets. 
Les crochets de la couronne externe mesurent 21-22 $\mu$ (fig. $2 a$ ), ceux de la couronne interne atteignent $23 \mu$.

Les ventouses dont l'orifice est très légèrement ovalaire sont relativement importantes et mesurent $220 \mu$ de diamètre et $120 \mu$ de profondeur. L'épaisseur du bourrelet muscu'aire marginal est de 12 à $15 \mu$. Celui-ci est armé de 3 à 4 ceintures de crochets dont les p'us longs insérés sur le bord antérieur mesurent 11 à $12 \mu$ (fig. $2 b$ ).

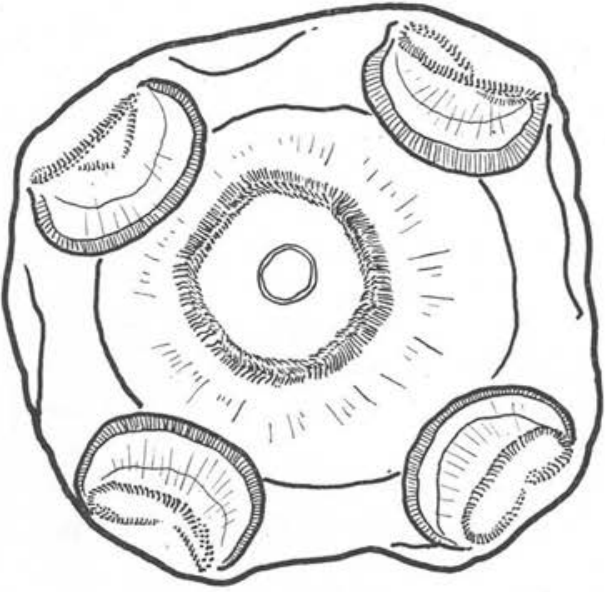

1

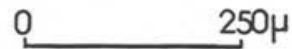

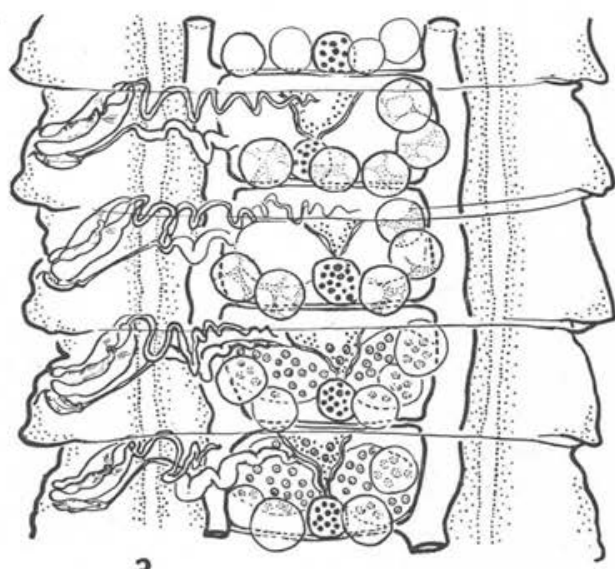

3

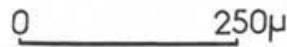

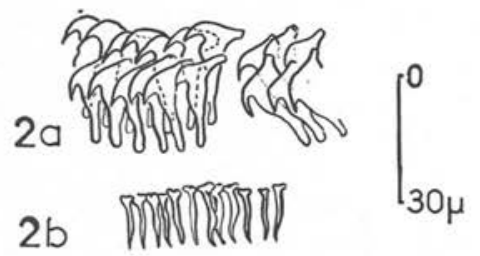

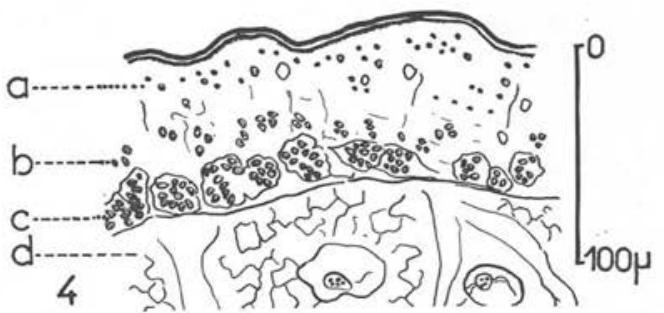

FIG. 1-4. - Raillietina (R.) khalili Hilmy 1936. - 1: scolex vue apicale. $-2 a$ : crochets du rostellum. $-2 b$ : crochets des ventouses. - 3: proglottis murs, face dorsale. -4 : couches musculaires sous-cutanées, coupe histologique transversale; $a$ ) fibres longitudinales isolées; $b$ ) faisceaux de deux à trois fibres; $c$ ) faisceaux de six à huit fibres ; $d$ ) parenchyme

\section{Cou.}

Très long et étroit, il atteint $2.800 \mu$ de longueur, pour une largeur de 170 à $180 \mu$, sur les échantillons non contractés.

\section{Appareil musculaire.}

Il est composé de trois couches superposées de fibres musculaires longitudinales (fig. 4). 
Une première couche sous-cuticulaire est représentée par quelques fibres isolées (fig. $4 a$ ).

Une seconde plus profonde est constituée par des faisceaux de 2 à 3 fibres (fig. $4 b$ ).

Enfin, une troisième couche plus importante aligne des faisceaux musculaires, serrés les uns contre les autres, de 6 à 10 fibres chacun (fig. $4 \mathrm{c}$ ). Ces faisceaux sont au nombre d'une vingtaine du côté dorsal, autant du côté ventral. Ils sont séparés de la région médullaire, où sont logés les organes génitaux, par une mince couche de fibres transversales.

\section{Système excréteur.}

Seuls sont visibles les deux canaux osmo-régulateurs ventraux, de 35 à $40 \mu$ de diamètre dans les anneaux mûrs.

Le canal osmo-régulateur ventral gauche passe sous les conduits génitaux. Au bord postérieur du proglottis, les canaux ventraux sont reliés entre eux par un canal osmorégulateur transversal de 3 à $5 \mu$ de diamètre. Les cordons nerveux longitudinaux sont ventraux par rapport aux conduits sexuels et externes, par rapport aux conduits osmorégulateurs.

\section{Proglottis (fig. 3).}

\section{A) MORPHOLOGIE GÉNÉrale ET DÉVELOPPEMENT :}

Les anneaux sont toujours plus larges que longs. La croissance des prog'ottis est très lente. La largeur n'augmente plus après le $600^{\circ}$ prog'ottis et décroît dans les tout derniers anneaux gravides.

Le tableau suivant rassemble l'évolution de l'appareil génital le long du strobile et quelques mensurations en $\mu$ des proglottis aux différentes étapes du développement.

Le premier tiers du strobile correspond à la formation des deux organes génitaux, le second à leur différenciation et maturation. Dans le troisième tiers après la fécondation, les œufs se rassemblent dans l'utérus. Celui-ci se cloisonne secondairement en capsules ovifères dans les proglottis gravides.

\begin{tabular}{|c|c|c|c|}
\hline $\mathrm{N}^{\circ}$ des proglottis & Largeur & Longueur & $\begin{array}{c}\text { Développement des organes } \\
\text { génitaux }\end{array}$ \\
\hline 250 & $420-430$ & 100 & ébauche des conduits génitaux. \\
\hline $260-370$ & $570-580$ & 100 & glande vitellogène et testicules \\
\hline 500 & 650 & $130-140$ & ovaire? origine difficile à déceler \\
\hline 600 & 980 & 130 & anneaux mûrs \\
\hline $640-670$ & 980 & 190 & l'utérus envahit le proglottis \\
\hline 840 & 1000 & 300 & $\begin{array}{l}\text { début de formation des capsules } \\
\text { ovifères }\end{array}$ \\
\hline 960 & 950 & 400 & derniers proglottis \\
\hline & 850 & 600 & gravides \\
\hline
\end{tabular}


L'épaisseur de la cuticule est de 2,5 à $3 \mu$.

B) APPAREIL REPRODUCTEUR :

\section{Atrium génital.}

Unilatéral, il est situé au milieu du bord du proglottis non contracté. Très légèrement saillant, son orifice annulaire est bordé par un bourrelet circulaire dont le diamètre mesure $13 \mu$.

2. Appareil reproducteur mâle.

a) Testicules. Au nombre très restreint de 4 ou 5 par anneau : 2 poraux et 2-3 antiporaux, les testicules sont sphériques ou légèrement ovalaires et présentent un diamètre de 50 à $60 \mu$ dans les anneaux mûrs. Ils occupent le bord supérieur du proglottis.

b) Canal déférent. Le canal déférent très contourné longe le bord antérieur du proglottis avant de pénétrer dans la poche du cirre ; son diamètre moyen est de 5 à $6 \mu$. Il n'est entouré d'aucun manchon de cellules glandulaires. Il n'existe pas de vésicule séminale externe.

c) Poche du cirré (fig. 5). Mesurant 140 à $170 \mu$ de long sur $60 \mu$ de large dans les anneaux mûrs, ovalaire ou piriforme, elle présente aussi une courbure dont la convexité est dirigée vers le bord postérieur du proglottis, son extrémité proximale étant la plus antérieure. La poche du cirre n'atteint jamais les canaux osmo-régulateurs. Le canal déférent se poursuit à l'intérieur de la poche du cirre par une vésicule séminale interne de 20 à $35 \mu$ de diamètre. Celle-ci communique avec le cirre invaginé par un canal de $3 \mu$ de diamètre, replié deux fois sur lui-même. Un faisceau de fibres radiales se rattache à l'extrémité du cirre invaginé. Le cirre n'a jamais pu être examiné évaginé, sa longueur est de $80 \mu$. Sa paroi épaisse de $1,5 \mu$ est tapissée intérieurement de soies longues de 10 à $12 \mu$. La paroi de la poche du cirre constituée de fibres musculaires circulaires est mince. Son épaisseur moyenne varie de 2 à $5 \mu$.

\section{Appareil reproducteur femelle.}

a) Vagin. Mesurant 70 à $75 \mu$ de long sur $15 \mu$ de large dans les anneaux sexuellement mûrs, il débouche dans l'atrium génital en arrière de la poche du cirre. Il est entouré de fibres musculaires particulièrement épaisses dans la région distale.

La lumière du canal vaginal varie de 3 à $5 \mu$ dans les anneaux mûrs. Le canal séminal très fin qui fait suite est pratiquement rectiligne sur $110 \mu$, jusqu'à l'extrémité de la poche du cirre. Il décrit 3 à 4 boucles avant d'aboutir près de la glande vitellogène. Sur son trajet long de $350 \mu$ environ, son diamètre très restreint au départ s'accroît et atteint 10 à $15 \mu$.

b) Ovaire. L'ovaire est bilobé, très peu colorable. Son origine est difficile à déceler le long du strobile. Dans les proglottis mûrs, de part et d'autre de l'ébauche de l'utérus, les deux lobes ovariens occupent tout l'espace compris entre les canaux osmo-régulateurs ventraux. Il n'a pas été possible sur nos échantillons de discerner les différents conduits génitaux femelles autour de la glande vitellogène. 
c) Glande vitellogène. Compacte, de forme presque toujours sphérique ou ovalaire, elle est ventrale derrière l'ovaire. Son diamètre est de $60 \mu$ environ dans les anneaux mûrs. Elle involue dans les premiers proglottis gravides. La glande de Mehlis n'est pas visible.
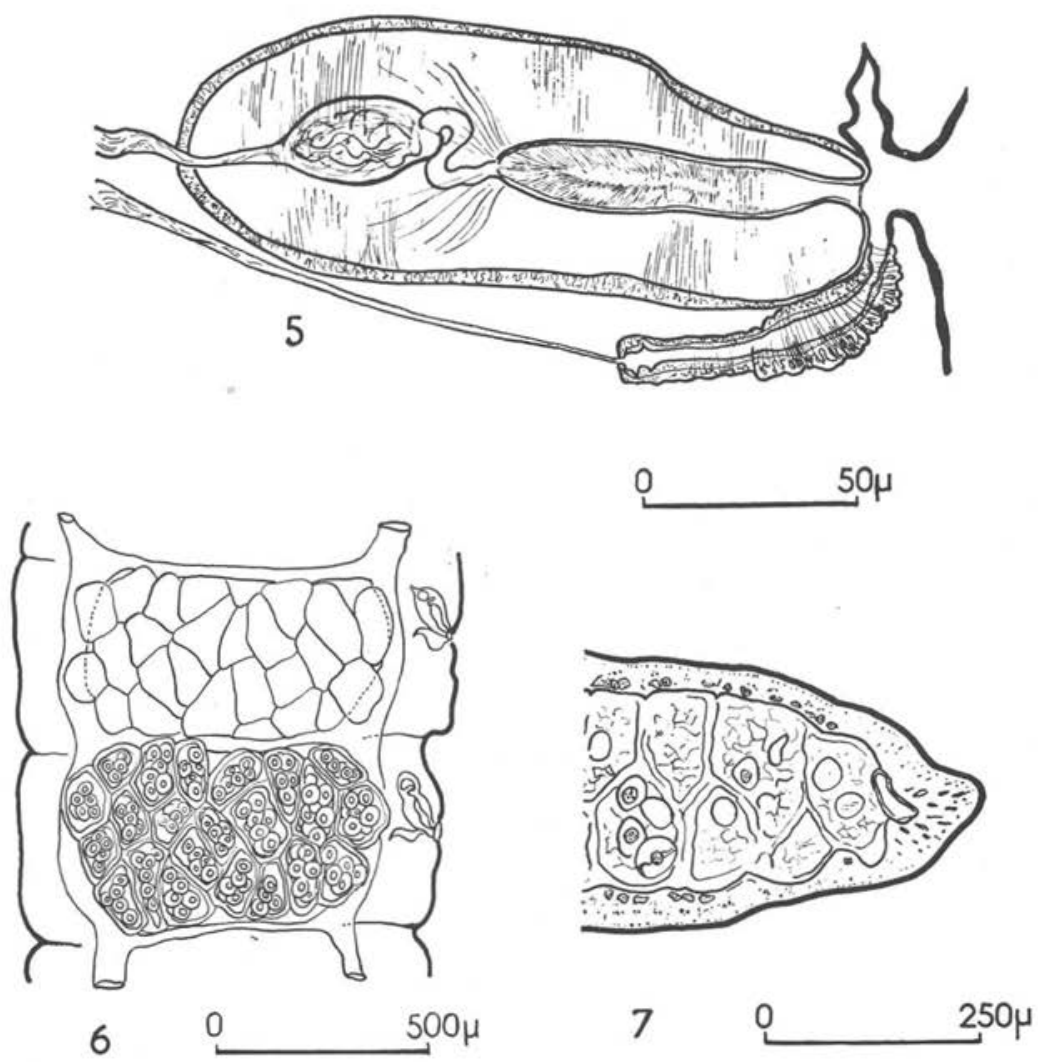

Fig. 5-7. - Raillietina $(R$.) khalili Hilmy 1936. - 5: détail de la poche du cirre et du vagin dans un proglottis mûr. - 6: Proglottis gravides. - 7: coupe histologique de la bordure marginale d'un proglottis montrant les deux champs superposés des capsules ovifères

d) Utérus (fig. 6). Son évolution est rapide (640-670 proglottis). Il débute par un petit sac situé dans la zone antérieure et dorsale du proglottis, à l'extrémité du canal utérin qui part au niveau de la glande vitellogène. Il s'accroît, écartant la zone parenchymateuse antérieure, occupant ensuite progressivement tout l'espace compris entre les canaux osmo-régulateurs. Les cloisons qui donneront naissance aux capsules ovifères apparaissent vers le $840^{\circ}$ proglottis. Les capsules sont disposées selon deux champs, l'un dorsal, l'autre ventral, de 22 à 23 capsules chacun (fig. 7), plus quelques capsules marginales, d'où un nombre total d'environ 45 à 49 capsules ovifères 
de 160 à $190 \mu$ de diamètre. Les six paires de crochets des embryons hexacanthes ne sont pas encore visibles.

En résumé, les caractères essentiels de ce Raillietina sont les suivants : Cestode de 12 à $13 \mathrm{~cm}$ de longueur ; nombre de proglottis : 900 ; largeur du scolex : 480-500 $\mu$, avec double couronne de crochets, au total : 280 à 300 crochets de 21-23 $\mu$; 4 ventouses armées de $220 \mu$ de diamètre. Les canaux osmo-régulateurs ventraux sont seuls visibles. Largeur des proglottis : 180-950 $\mu$, longueur des proglottis : 100-600 $\mu$; 4 à 5 testicules par proglottis; poche du cirre: 140-170 $\mu$ de long sur $60 \mu$ de large, diamètre de la vésicule séminale : $35 \mu$; vagin : 70 à $75 \mu$ de long; environ 50 capsules ovifères par proglottis contenant chacune 8 à 10 œufs mesurant 40 à $45 \mu$ de diamètre.

\section{2) DISCUSSION :}

Cinq espèces appartenant au sous-genre Raillietina (Raillietina) sont parasites de Cuculiformes. Ce sont :

- R. (R.) calcaria (Fuhrmann 1909, p. 109, fig. 18-19), chez Corythaeola cristata (Vieillot) et Centropus monachus monachus Ruppell, Afrique.

- R. (R.) khalili Hilmy 1936 , p. 24-26, pl. III, fig. 5-6, chez Turacus persa buffoni (Vieillot), Libéria.

- R. (R.) macrocirrosa (Fuhrmann 1909, p. 110, fig. 20), chez Turacus persa buffoni (Vieillot), Centropus senegalensis senegalensis (Linné) et $C$. monachus monachus Ruppell, Afrique.

- R. (R.) turaci Baer 1933, p. 65-69, chez Turacus livingstonii loitanus (Neumann), Afrique.

- R. (R.) undulata (Fuhrmann 1909, p. 109-110), chez Corythaeola cristata (Vieillot), Chrysococcyx et Gallirex sp., Afrique.

Parmi ces cinq espèces, seules $R$. (R.) undulata (Fuhrmann 1909) et $R$. (R.) khalili Hilmy 1936 possèdent des crochets d'une taille supérieure à $20 \mu$. Nous écartons $R$. (R.) undulata, dont le nombre total de crochets est insuffisant : 200, pour pouvoir être comparé à celui de l'espèce étudiée, et qui possède en outre un nombre trop élevé de testicules : 12 à 15 d'après Fuhrmann 1909, p. 109-110, 20 d'après Baer 1936, p. 65.

$R$. (R.) khalili Hilmy 1936, p. 24-26, pl. III, p. 5-6, présente par contre de nombreuses affinités morphologiques et anatomiques avec le Cestode étudié. Cette espèce possède en effet un scolex large de $460 \mu$ dont le rostellum invaginé porte une double couronne de 300 crochets de $21 \mu$ de long. Les détails de l'anatomie génitale et de la musculature correspondent à notre description. La poche du cirre mesure $172 \mu$ de long sur $73 \mu$ de large. Le diamètre de la vésicule séminale interne est de $32 \mu$. Les testicules sont plus nombreux (7 à 11) que chez notre espèce où nous n'avons compté que 4 à 5 testicules par proglottis. Les anneaux gravides de $R$. (R.) khalili Hilmy 1936 portent chacun 60 capsules ovifères comprises entre les canaux osmo-régulateurs, nombre légèrement supérieur à celui de nos échantillons. La seule différence dans le nombre de 
testicules ne constitue pas à notre point de vue un élément valable pour la création d'une espèce nouvelle et nous pensons que le Cestode étudié peut s'identifier à $R$. $(R$.) khalili décrit par Hilmy en 1936.

\section{RAILLIETINA (RAILLIETINA) DOUCETI n. sp.}

MATÉRIEl Étudié: une dizaine d'individus dont certains avec proglottis gravides, de Turacus sp. (Cuculiforme), J.-P. Doucet, leg., 22 janvier 1955, Côte-d'Ivoire.

\section{1) DESCRIPTION :}

La longueur des Cestodes varie de 6 à $12 \mathrm{~cm}$ selon le degré de contraction du strobile. Nous avons compté 700 à 800 proglottis chez les vers les plus complets dont les derniers anneaux gravides sont remplis de capsules ovifères.

1. Scolex (fig. 8-9).

Bien différencié du strobile, d'une largeur d'environ $500 \mu(480-550 \mu)$, il présente une hauteur de $350 \mu$ environ (330-390 $\mu$ ).

Le rostellum est resté invaginé sur tous les scolex observés. Etudiée en vue apicale et bien étalée, sa doub'e couronne de crochets présente un diamètre interne de 150 à $170 \mu$. Le nombre total de crochets est d'environ 270 , nombre constant à 2-3 crochets près. Les crochets de la couronne externe mesurent $15 \mu$, ceux de la couronne interne $16 \mu$ (fig. $10 a$ ).

Les ventouses sphériques ont un diamètre de 180 à $200 \mu$. L'orifice de chaque ventouse mesure 120 à $150 \mu$ de diamètre, il est bordé d'un bourrelet marginal armé de 3 à 4 ceintures de crochets dont les plus longs mesurent $15 \mu$ (fig. $10 \mathrm{~b}$ ). La taille et la densité de ces crochets diminuent progressivement de l'avant vers l'arrière de la ventouse où l'on n'observe plus qu'une seule ceinture.

\section{Cou.}

Sa longueur sur les échantillons non contractés atteint 1.200 à $1.300 \mu$ pour une largeur de 200 à $300 \mu$.

\section{Appareil musculaire (fig. 11).}

Peu important chez cette espèce, il est constitué de fibres musculaires longitudinales, d'abord isolées et très peu nombreuses sous la zone sous-cuticulaire (fig. 11 a) caractérisée par de nombreux noyaux. Une deuxième couche plus profonde de faisceaux, de 2 à 3 fibres chacun (fig. $11 \mathrm{~b}$ ), se distingue mal d'une troisième groupant principalement des faisceaux de 4 fibres environ (fig. $11 \mathrm{c}$ ). La faible densité des fibres musculaires rend ici la zonation plus diffuse.

\section{Système excréteur.}

Il est composé de deux paires de canaux osmo-régulateurs longitudinaux au tracé sinueux (fig. 12). Les deux canaux dorsaux étroits (diamètre : 5 à $6 \mu$ ) ne sont visibles 

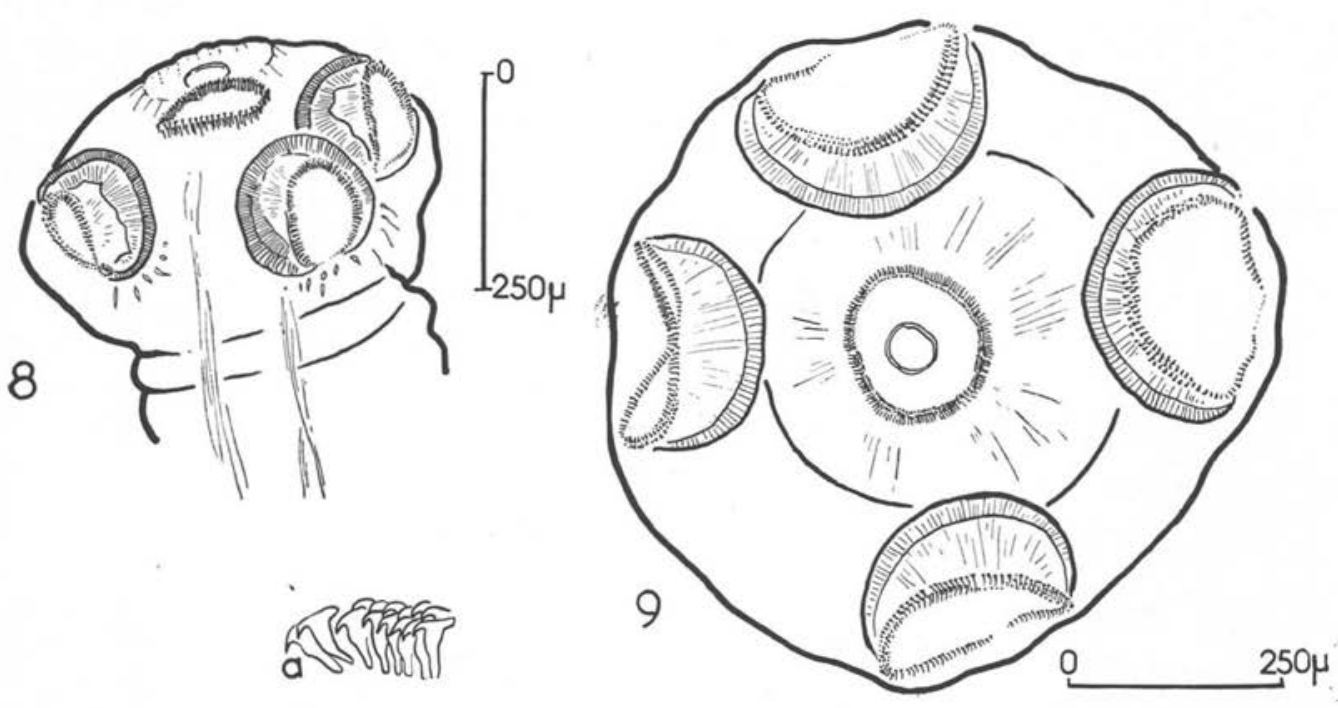

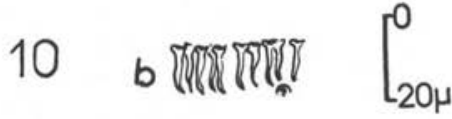
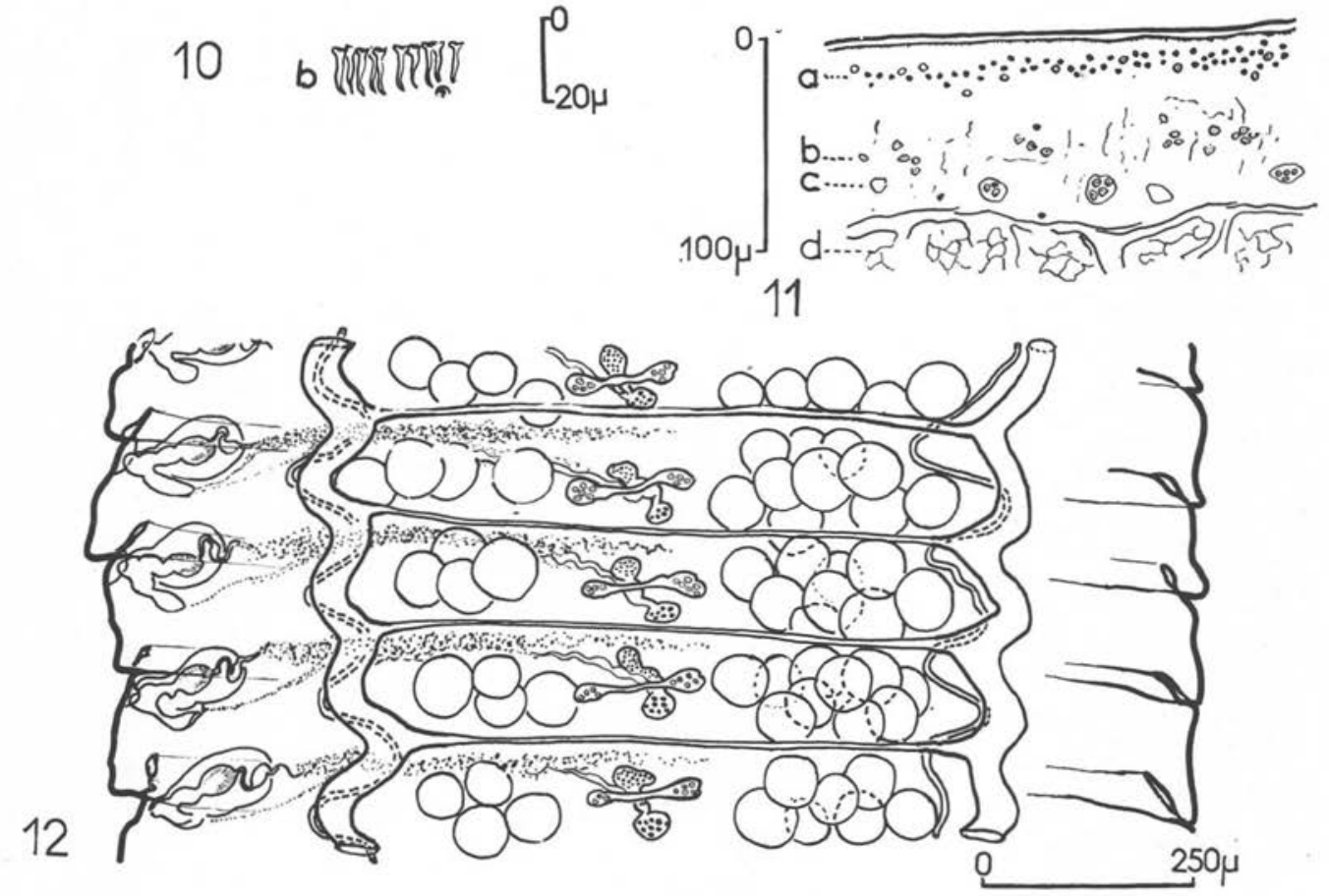

FIG. 8-12. - Raillietina $(R$.) douceti. n. sp. -8 : scolex vue semi-latérale. - 9: scolex vue apicale. $-10: a)$ crochets du rostellum. $-10: b)$ crochets des ventouses. - 11: couches musculaires sous-cutanées, coupe histologique transversale; $a$ ) fibres longitudinales isolées; $b$ ) faisceaux de deux à trois fibres chacun; $c$ ) faisceaux de quatre fibres; $d$ ) parenchyme. -12 : proglottis jeunes possédant deux paires de canaux osmo-régulateurs, vue ventrale 
que dans les proglottis immatures. Les deux canaux ventraux (diamètre : 25 à $30 \mu$ dans les anneaux jeunes) sont reliés à la partie postérieure du proglottis par un canal osmo-régulateur transversal de $12 \mu$ de diamètre.

Les conduits génitaux passent entre les canaux osmo-régulateurs. A mesure que l'on progresse le long du strobile, les canaux osmo-régulateurs ventraux s'élargissent. Leur diamètre augmente rapidement : 150 à $200 \mu$ dans les anneaux gravides. Il diminue dans les derniers anneaux de la chaîne : 70 à $80 \mu$.

5. Proglottis (fig. 13).

A) Morphologie générale et développement :

De forme trapézoïdale, les anneaux sont toujours plus larges que longs. La largeur augmente progressivement le long du strobile, atteint son maximum dans les premiers proglottis gravides (largeur : 1.400 ; longueur : 1.750), puis décroît dans les derniers proglottis remplis de capsules ovifères. A maturité, les proglottis relativement bien étendus mesurent $1.100 \mu$ de largeur sur 300 à $400 \mu$ de longueur.

La cuticule lisse est épaisse de $4 \mu$.

L'évolution de l'appareil génital le long du strobile est résumée dans le tableau suivant.

\begin{tabular}{|c|c|}
\hline $\mathbf{N}^{\circ}$ des proglottis & $\begin{array}{l}\text { Etapes du développement } \\
\text { des organes génitaux }\end{array}$ \\
\hline 400 & - premières ébauches des conduits génitaux. \\
\hline 430 & $\begin{array}{l}\text { - apparition des testicules, ébauches de la poche du cirre } \\
\text { et de la glande vitellogène. }\end{array}$ \\
\hline 500 & $\begin{array}{l}\text { - conduits génitaux, poche du cirre et glande vitellogène } \\
\text { bien différenciés. }\end{array}$ \\
\hline 520 & — ébauche de l'utérus. \\
\hline 530 & — ébauche de l'ovaire. \\
\hline $550-600$ & — maturité des organes génitaux. \\
\hline 620 & $\begin{array}{l}\text { - l'utérus envahit l'espace compris entre les canaux osmo- } \\
\text { régulateurs (fig. 14), régression des organes reproduc- } \\
\text { teurs à l'exception des testicules. }\end{array}$ \\
\hline 670 & — cloisonnement de l'utérus en capsules ovifères. \\
\hline 700 & - capsules ovifères différenciées (fig. 16). \\
\hline
\end{tabular}

B) Appareil reproducteur :

\section{Atrium génital.}

Unilatéral, il est situé au milieu du bord marginal des proglottis normalement étendus. Légèrement saillant, il présente un orifice dont le diamètre mesure 70 à $80 \mu$. Le vagin débouche en arrière de la poche du cirre, légèrement en retrait par rapport à l'extrémité de celle-ci. 

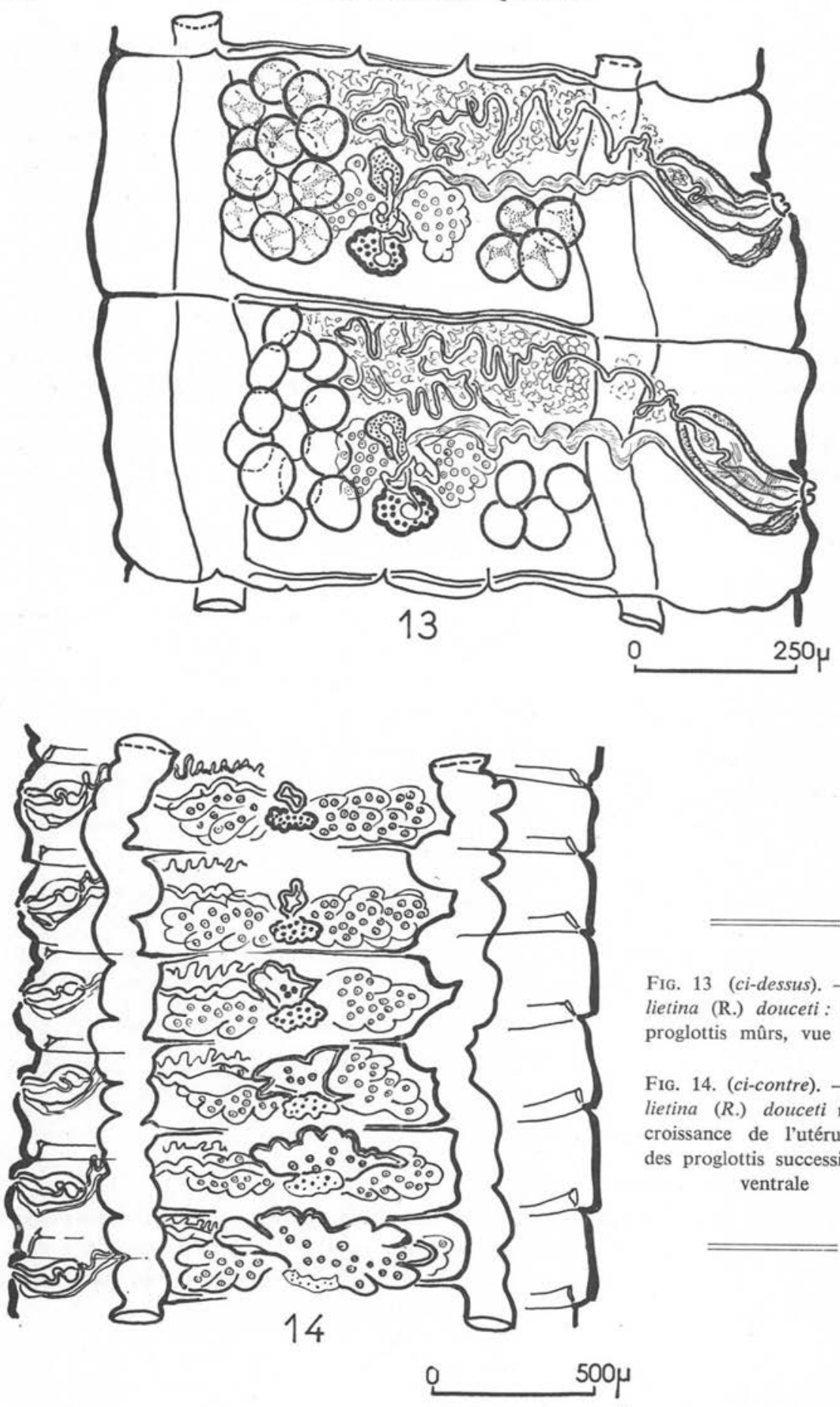

FIg. 13 (ci-dessus). - Raillietina (R.) douceti : n. sp. : proglottis mûrs, vue dorsale

FIG. 14. (ci-contre), - Raillietina $(R$.) douceti $\mathrm{n}$. sp.: croissance de l'utérus dans des proglottis successifs, vue ventrale 


\section{Appareil reproducteur mâle.}

a) Testicules. Au nombre de 15 à 18 par proglottis, légèrement ovalaires, d'un diamètre de 70 à $80 \mu$ dans les anneaux mûrs, ils sont disposés en deux groupes de part et d'autre des organes génitaux femelles : 3 à 4 testicules poraux et 12 à 14 testicules antiporaux. Ils ne dépassent pas latéra'ement la limite constituée par les canaux osmorégulateurs. Les plus antérieurs dépassent le niveau du sommet de l'utérus. Il n'existe pas de testicules à l'arrière de la glande vitellogène.

b) Canal déférent. Il chemine dorsalement dans la région antérieure de chaque proglottis. Fortement contourné, il est entouré d'un manchon épais de cellules glandulaires très turgescentes. Son diamètre avant d'aboutir à la poche du cirre est de 10 à $11 \mu$.

Il n'existe pas de vésicule séminale externe.

c) Poche du cirre (fig. 17). De taille relativement importante, ovale dans les proglottis immatures, elle atteint à ce niveau $170 \mu$ de long sur $65 \mu$ de large. Piriforme dans les anneaux sexuellement mûrs, elle mesure 210 à $240 \mu$ de long sur 90 à $100 \mu$ de large. Elle présente alors une courbure dont la convexité est dirigée vers le bord postérieur du proglottis, son extrémité proximale étant la plus antérieure. Elle n'atteint jamais les canaux osmo-régulateurs. Sa paroi musculeuse est épaisse de 10 à $14 \mu$ dans la région moyenne de l'organe, elle est constituée de fibres musculaires circulaires.

Le canal déférent se poursuit à l'intérieur de la poche du cirre par une vésicule séminale interne, ovale, de $40 \mu$ de longueur. Elle peut atteindre $60 \mu$ de diamètre à son maximum de volume. Fait suite un canal replié deux fois sur lui-même, de 3 à $4 \mu$ de diamètre, qui relie la vésicule séminale interne au cirre invaginé. Celui-ci n'a jamais pu être observé évaginé. A l'intérieur de la poche du cirre dans les proglottis mûrs, il mesure $100 \mu$ de long sur 15 à $23 \mu$ de large. Sa paroi de 1,5 à $2 \mu$ d'épaisseur est tapissée intérieurement de fines soies longues de 13 à $14 \mu$. Leur longueur diminue vers la région distale de la poche du cirre, les soies sont alors à peine visibles.

La poche du cirre a une morphologie comparable à celle de $R$. (R.) khalili Hilmy 1936, trouvé chez le même hôte. Elle se caractérise cependant, d'une part, par sa taille qui est une fois et demie plus importante que celle de la poche du cirre de l'espèce déjà décrite, d'autre part, par sa paroi qui est ici environ trois fois plus épaisse.

\section{Appareil reproducteur femelle.}

a) Vagin. Mesurant 100 à $110 \mu$ de long sur 20 à $30 \mu$ de diamètre dans les segments sexuellement mûrs, il débouche dans l'atrium génital en arrière et en aval de la poche du cirre. Il est entouré d'un manchon de fibres musculaires particulièrement épais dans la moitié distale du vagin. La lumière du canal vaginal varie de quelques microns à $15 \mu$ selon le degré de maturité sexuelle, elle est tapissée de très fines soies. Le vagin se poursuit par un canal séminal fin et rectiligne sur $170 \mu$ jusqu'à l'extrémité proximale de la poche du cirre. Le diamètre du canal séminal augmente consịdérablement au niveau des canaux osmo-régulateurs et, après les avoir franchis, il atteint alors 30 à $40 \mu$ de large. La longueur totale du canal séminal est de $600 \mu$ environ dans les 

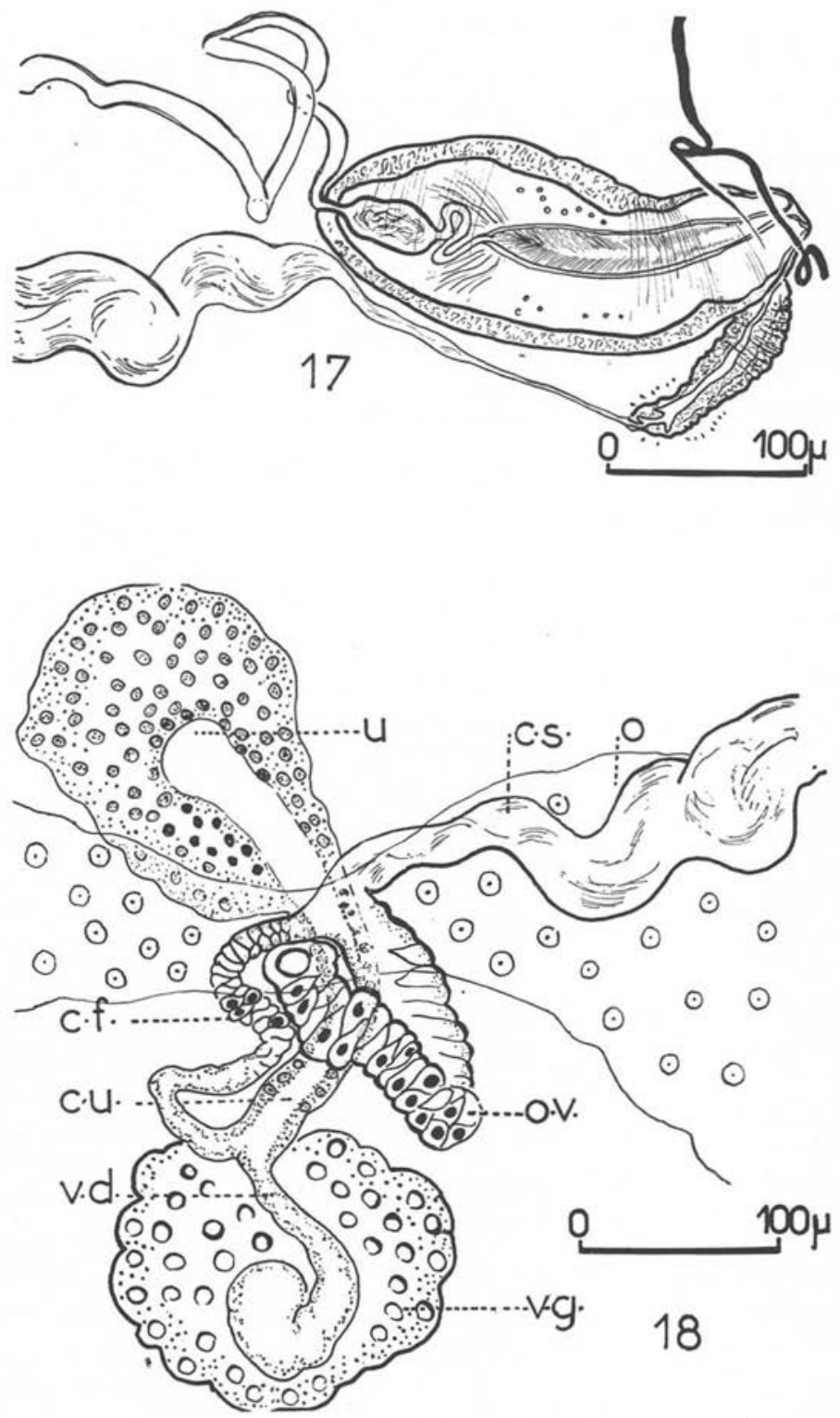

FIG. 17-18. - Raillietina $(R$.) douceti $\mathrm{n}$. sp. -17 : détail de la poche du cirre et du vagin dans un proglottis mûr. -18 : conduits génitaux femelles; c.f. : canal de fertilisation, c.s. : canal séminal, c.u. : canal utérin, o. : ovaire, o.v. : oviducte, u. : utérus, v.d. : vitelloducte, v.g. : glande vitellogène. 
anneaux mûrs où il joue le rôle d'un réceptac'e séminal. Le canal séminal franchit dorsalement l'isthme ovarien et s'abouche à l'oviducte.

b) Ovaire. Bilobé, l'ovaire est sensiblement décalé du côté antiporal et présente en outre une légère obliquité dirigée vers l'atrium. L'oviducte naît approximativement au centre de l'isthme ovarien (fig. 18). Il décrit une première boucle (diamètre : 20-25 $\mu$ ) à l'extrémité de laquelle il reçoit le canal séminal ; il se contourne alors sur lui-même pour constituer une seconde boucle presque perpendiculaire à la première et qui représente le canal de fertilisation (diamètre : 12 à $15 \mu$ ). La paroi de l'oviducte est garnie de grosses cellules glandulaires. Le canal de fertilisation se contourne à son tour en diminuant de diamètre tout en revenant vers la glande vitellogène dont il reçoit un mince vitelloducte large d'une dizaine de $\mu$ et long d'environ $100 \mu$. Il se poursuit enfin par le canal utérin légèrement courbé, de calibre régulier $(25 \mu)$, et d'orientation grossièrement postéro-antérieure qui vient croiser l'isthme ovarien pour former l'utérus proprement dit.

c) Glande vitellogène. Compacte, de forme variable, elle est ventrale derrière l'ovaire. Son diamètre est de $120 \mu$ dans les anneaux mûrs (valeurs extrêmes : 80-170 $\mu$ ). L'accroissement de volume de la glande vitellogène dans les premiers prog!ottis gravides précède sa disparition.

La glande de Mehlis est peu visible et mal délimitée.

d) Utérus. Visible dès la formation du complexe génital femelle, il apparaît d'abord comme une petite masse cellulaire compacte, située antérieurement par rapport à la glande vitellogène et les ovaires, et dorsalement à ceux-ci. Sa forme est variable dans les prog!ottis mûrs, mais on distingue presque toujours le canal utérin. Il augmente de volume à partir du $620^{\circ}$ prog'ottis et occupe celui-ci en entier vers le $640^{\circ}$. Les capsules ovifères issues du cloisonnement de l'utérus, très nettement différenciées à partir du $700^{\circ}$ proglottis, sont disposées en deux champs superposés dont chacun groupe 50 à 60 capsules comprises entre les canaux osmo-régulateurs (fig. 15-16).

Le nombre total de capsules ovifères peut donc être évalué entre 100 et 120 capsules, chacune d'elles renferme 6 à 8 œufs. Les œufs réhydratés dans l'eau physiologique sont sphériques et mesurent 40 à $45 \mu$ de diamètre.

L'oncosphère ellipsoïdale de $12-16 \times 20-27 \mu$ est recouverte d'une coque interne. Les proglottis gravides examinés ne renfermaient pas d'embryon hexacanthe bien différencié. Il n'a pas été possible de discerner les trois paires de crochets et de les mesurer.

En résumé, les caractères essentiels de ce Raillietina sont les suivants : longueur : $12 \mathrm{~cm}$ chez les exemplaires les plus complets, 800 proglottis. Largeur du scolex : $500 \mu$; rostre avec double couronne de crochets : au total 270 crochets de 15 et $16 \mu$; 4 ventouses armées de 180 à $200 \mu$ de diamètre ; 2 paires de canaux osmo-régulateurs ; largeur des proglottis : $300-1.400 \mu$; longueur : $100-450 \mu ; 15$ à 18 testicules ; poche du cirre : $210-240 \mu$ de long sur $90-100 \mu$ de large ; vagin : $100-110 \mu$ de long; 100 à 120 capsules ovifères par prog'ottis contenant chacune 6 à 8 œufs de 40 à $45 \mu$ de diamètre. 


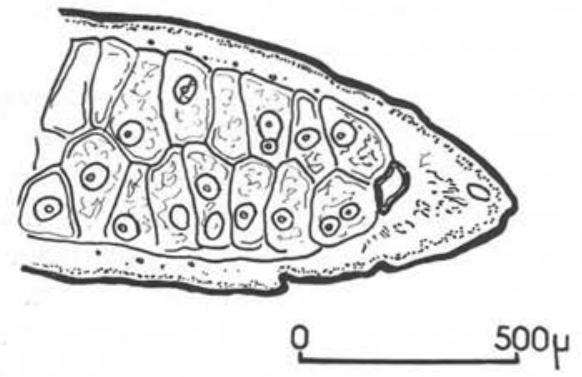

FIG. 15. - Raillietina (R.) douceti n. sp.: coupe transversale de la bordure marginale d'un proglottis gravide montrant les deux champs superposés de capsules ovifères.

\section{5}

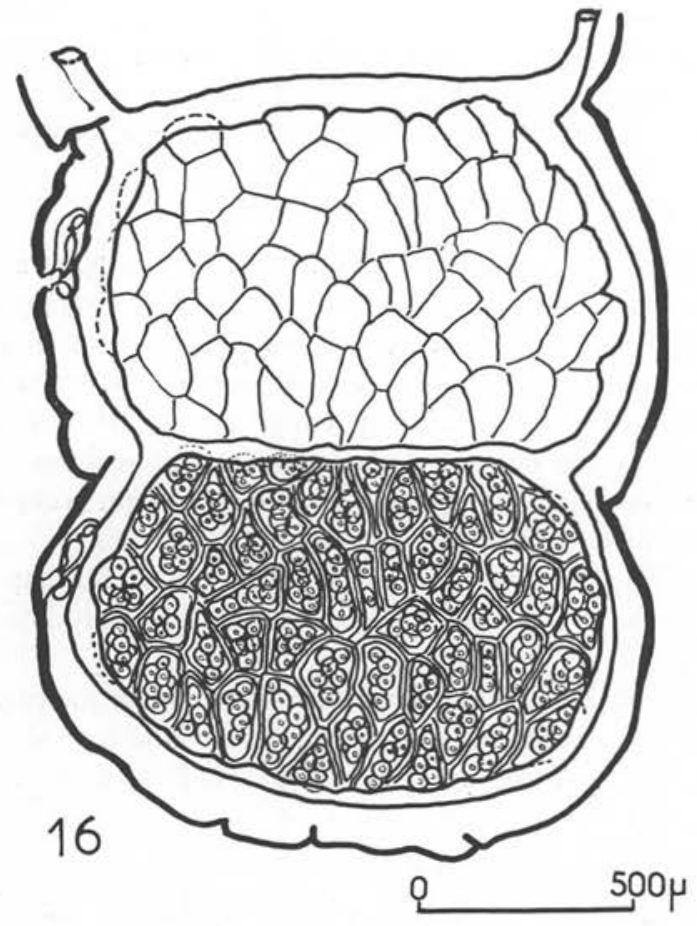

FIG. 16. - Raillietina (R.) douceti n. sp. : proglottis gravides, capsules ovifères comprises entre les canaux osmo-régulateurs.

\section{2) DISCUSSION :}

Cinq espèces appartenant au sous-genre Raillietina ont été décrites jusqu'à présent chez les Cuculiformes. De ces cinq espèces, deux seulement présentent les crochets de la double couronne de nombre et de dimensions sensiblement voisins de ceux des échantillons étudiés ; il s'agit de : 
R. (R.) calcaria (Fuhrmann 1909) qui possède d'après Fuhrmann 300 crochets de 14 et $16 \mu$ et, d'après Baer 1925,320 crochets de 16 à $17 \mu$, et de $R$. $(R$.) macrocirrosa (Fuhrmann 1909) qui possède 275 à 300 crochets de 13 à $14 \mu$.

Notre espèce (voir ci-dessus les caractères essentiels) possède donc un nombre total de crochets inférieur à celui de $R$. $(R$.) calcaria, et des crochets de dimensions supérieures à celles des crochets de $R$. (R.) macrocirrosa. En outre, elle se distingue de $R$. (R.) macrocirrosa par l'absence de gros muscle sphincter entourant l'atrium génital et de l'espèce calcaria, en premier lieu par le nombre plus élevé de testicules (18 contre 14 ) et, en second lieu, par la longueur beaucoup plus importante de la poche du cirre (210-240 contre $140 \mu)$.

Compte tenu de ces différences, nous considérons notre espèce comme nouvelle et sommes heureux de la dédier à J.-P. Doucet qui l'a récoltée.

\section{RAILLIETINA (RAILLIETINA) ORTLEPPI n. sp.}

MATÉRIEl ÉtUdié : nombreux Cestodes ne comportant pas encore de proglottis gravides, plus de fragments de strobiles à différents stades de maturité sexuelle, récoltés chez Vinago waahli (Columbiforme), originaire d'Afrique, ayant séjourné en captivité au Zoo de Milan, et mort à la ménagerie du Jardin des Plantes à Paris, le 29 novembre 1962. (Matériel aimablement communiqué par notre collègue Mme A. Petter).

\section{1) DESCRIPTION :}

Cestodes de petites dimensions, les strobiles les plus longs mesurent 15 à $20 \mathrm{~mm}$ de long pour une largeur maxima de $1 \mathrm{~mm}$ (anneau en début de gravidité). Il est par conséquent impossible de connaître le nombre de proglottis constituant un strobile entier possédant des anneaux gravides. L'exemplaire comportant le plus grand nombre de proglottis en détient 250 et les derniers proglottis présentent tout au plus un début de formation des capsules ovifères.

1. Scolex (fig. 19).

Bien différencié du strobile, large de 250 à $285 \mu$, il porte un rostre avec double couronne de crochets et quatre ventouses armées.

Sur des scolex appartenant à différents Cestodes, nous avons compté successivement : 340-360-400-424 crochets longs de 13 et $14 \mu$. Le diamètre de la double couronne varie selon que le rostre est invaginé ou non :

— rostre évaginé avec couronne bien étalée : $150 \mu$ de diamètre ;

— rostre évaginé, couronne plus contractée : $120 \mu$;

— rostre invaginé : $75 \mu$.

Le rostre est recouvert de très fines épines. Ce détail retrouvé chez Raillietina $(R$.) columbiella Ortlepp 1938 , p. 53, R. (R.) idiogenoides Baer 1933, p. 61, et $R$. (R.) torquata (Meggitt 1924), p. 307-309, fig. 3, n'est visible que lorsque le rostre est évaginé. 
Les quatre ventouses, ovales $(80-85 \times 50-65 \mu)$, ont une bordure musculaire armée de sept à huit rangées de crochets dans la partie antérieure. Les crochets des ventouses mesurent 12 à $13 \mu$.

\section{Cou.}

Sa longueur est de $700 \mu$. Sa largeur atteint $110 \mu$.

3. Appareil musculaire (fig. 20).

La musculature se différencie progressivement le long du strobile, parcouru en épaisseur par trois zones de fibres musculaires longitudinales :

- une couche de fibres sous-cuticulaires isolées;

- une deuxième couche composée de fibres groupées par deux à trois ;

- une troisième, plus profonde, formée de faisceaux de cinq à six fibres chacun, délimite la région médullaire.

Il existe 12 à 14 faisceaux du côté dorsal, autant du côté ventral.

\section{Appareil excréteur.}

Il est constitué de deux paires de canaux osmorégulateurs longitudinaux superposés. Sur le côté gauche du strobile, le canal osmorégulateur ventral passe sous les conduits génitaux, le canal osmorégulateur dorsal passe au-dessus. Les canaux dorsaux ont un diamètre de $2,5 \mu$. Les canaux ventraux, de calibre plus important (15 à $25 \mu$ de diamètre), sont reliés à la partie postérieure du proglottis par un canal transversal de 7 à $8 \mu$ de diamètre.

\section{Proglottis (fig. 21).}

\section{A) Morphologie gÉnÉrale et dÉveloppement :}

Les proglottis sont trapézoïdaux, plus larges que longs. Au fur et à mesure que l'on progresse le long du strobile, la croissance en longueur est proportionnellement plus importante qu'en largeur. Cependant, les dimensions ne s'égalisent jamais, même dans les proglottis gravides qui, remplis d'une dizaine de capsules ovifères, sont bombés latéralement. La cuticule épaisse, de 1,5 à $3 \mu$, est lisse.

Les mesures en $\mu$ des proglottis aux différentes étapes de leur développement sont rapportées dans le tableau suivant :

\begin{tabular}{||l|c|c||}
\hline \hline & Longueur & Largeur \\
\cline { 1 - 2 } Premiers proglottis & $15-20$ & 125 \\
$100^{\circ}$ & $110-120$ & 230 \\
proglottis mûrs & $270-280$ & 600 \\
proglottis gravides: & & 660 \\
- début de gravidité & 360 & 640 \\
\hline - gravidité moyenne & 490 & \\
\hline \hline
\end{tabular}




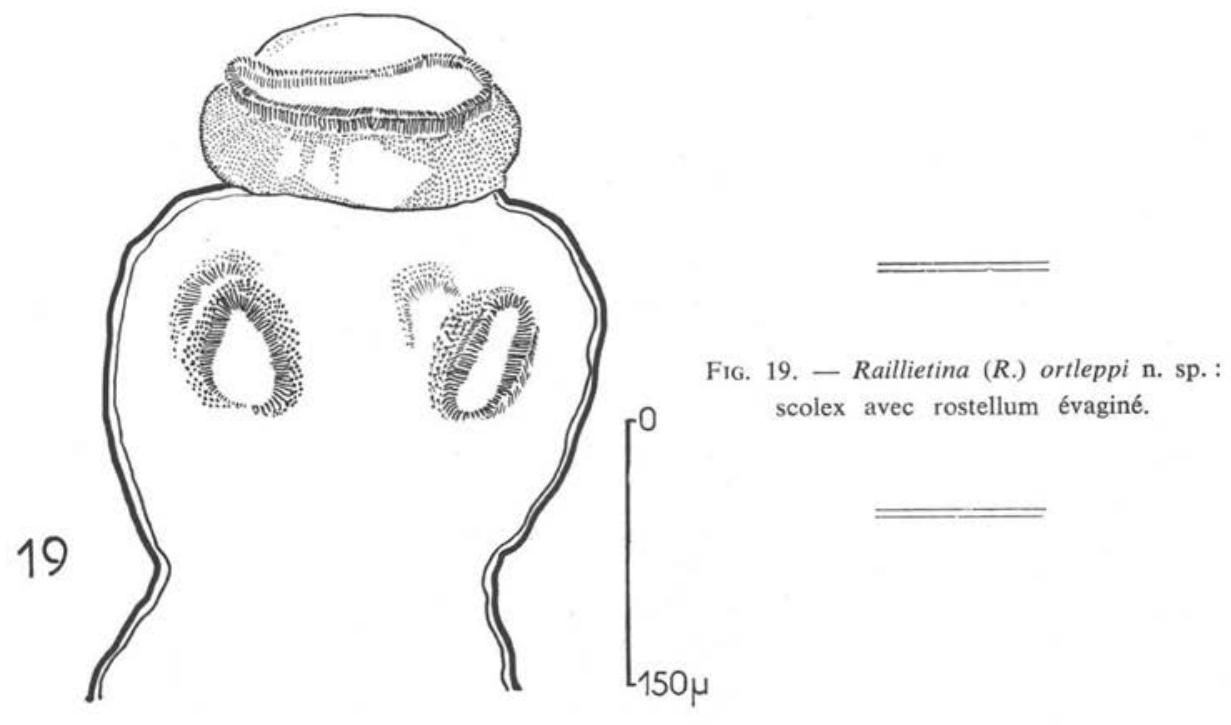

F1G. 20. - Raillietina (R.) ortleppi n. sp.: coupe histologique transversale oblique, passant par la poche du cirre et la glande vitellogène, détail de la paroi musculaire.

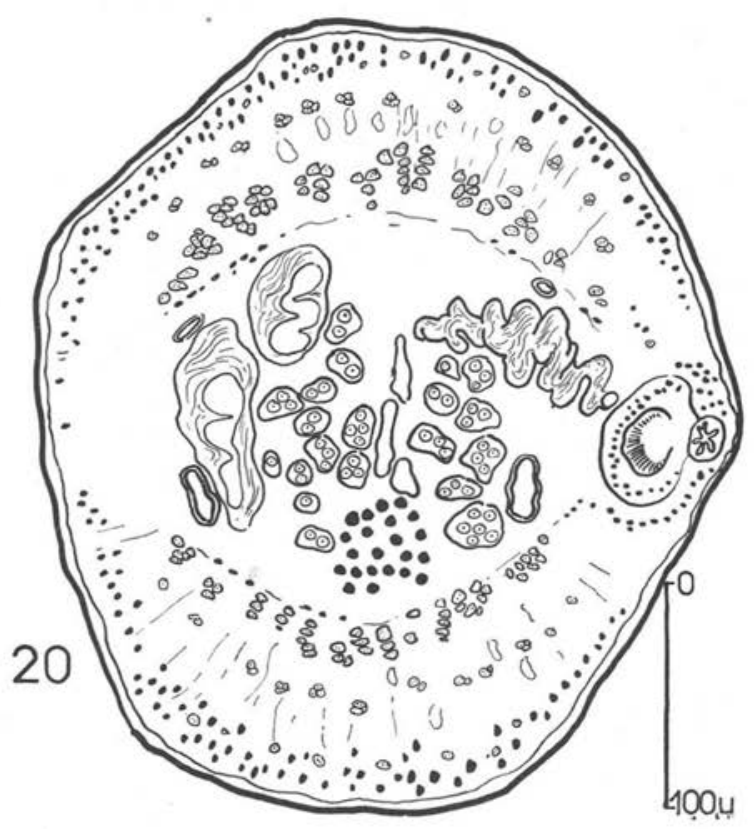

Les premières ébauches de l'atrium génital apparaissent vers le $120^{\circ}$ proglottis. Celles des testicules ne sont visibles qu'à partir du $150^{\circ}$, devançant l'apparition de l'ovaire qui ne se développe qu'après le $190^{\circ}$ proglottis. 

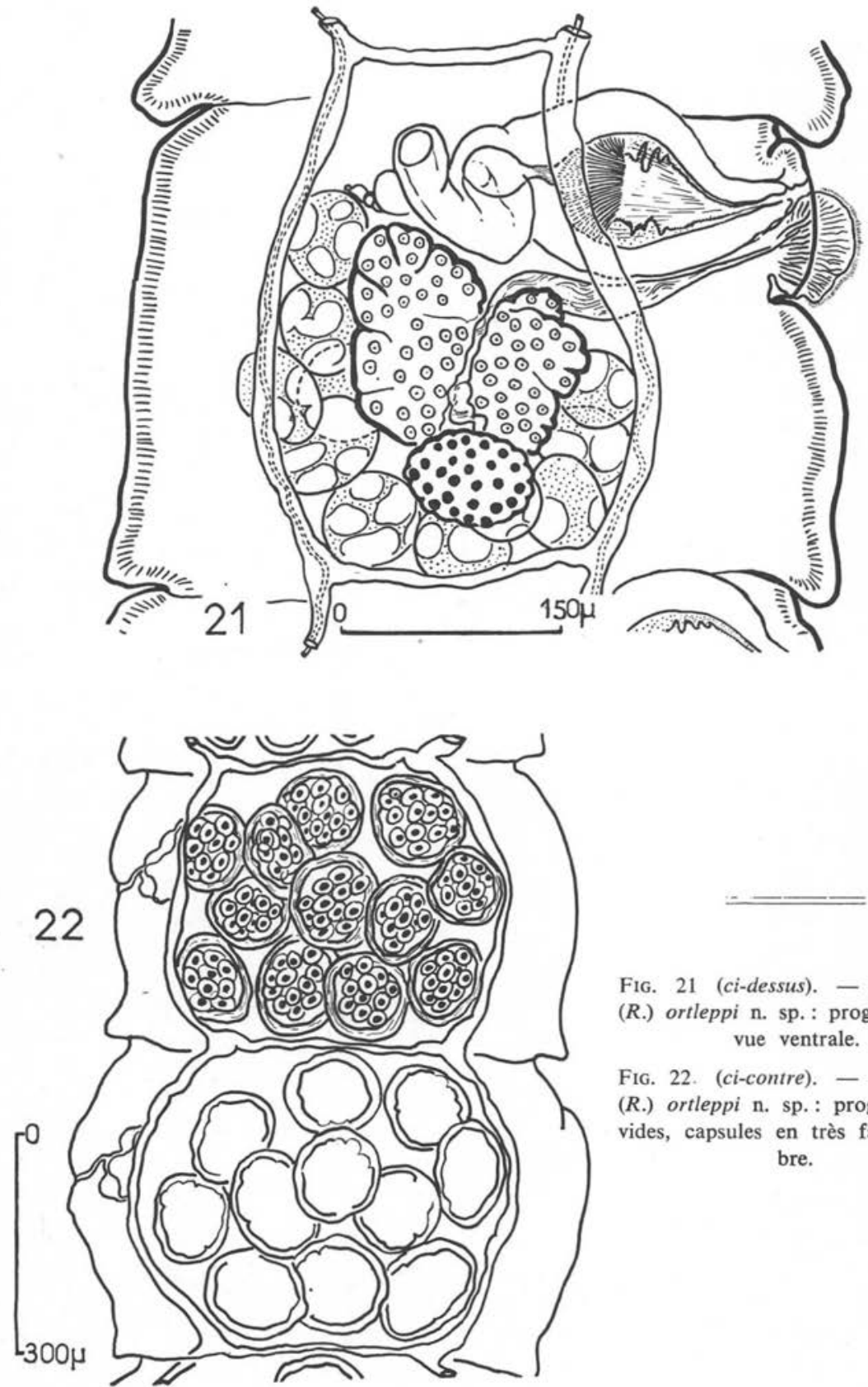

FIG. 21 (ci-dessus), - Raillietina (R.) ortleppi n. sp. : proglottis mûr, vue ventrale.

FIG. 22. (ci-contre). - Raillietina (R.) ortleppi $\mathrm{n}$. sp.: proglottis gravides, capsules en très faible nombre. 


\section{B) ApPareIl REPRODUCTEUR:}

\section{Atrium génital.}

Les pores génitaux sont unilatéraux, situés à la limite du $1^{\mathrm{er}}$ et du $2^{\circ}$ quart antérieur du proglottis.

En forme de cupule, l'atrium génital est de dimensions re'ativement constantes. Le diamètre du bourrelet qui entoure les orifices génitaux est de 90 à $100 \mu$. La profondeur de l'atrium (bord du proglottis jusqu'à la poche du cirre) atteint 45 à $50 \mu$. Le bourrelet est constitué de fibres musculaires radia'es ramifiées vers la périphérie, et d'une condensation cellulaire sous-cuticulaire. La cuticule de la cavité atriale est inerme.

\section{Appareil reproducteur mâle.}

a) Testicules. Ils sont disposés entre les canaux osmorégulateurs occupant les trois quarts postérieurs du proglottis. Le champ testiculaire enveloppe la région postérieure du complexe génital femelle. Environ, un tiers des testicules occupent le côté poral, deux tiers sont antiporaux. Le nombre total de testicules par proglottis est en moyenne de 16 à 18 (extrêmes : 12-18). Sphériques ou ova'aires, les testicules ont un diamètre de 50 à $60 \mu$ dans les anneaux sexuellement mûrs. Apparus dès le $150^{\circ}$ proglottis, ils persistent très longtemps dans les proglottis gravides.

b) Canal déférent. Situé dans la région antérieure du prog'ottis, il effectue quatre à cinq boucles serrées avant d'atteindre la poche du cirre.

Dans les anneaux jeunes $\left(180-190^{\circ}\right)$, son diamètre moyen est de $5 \mu$. Ce calibre s'accroît rapidement dans les anneaux mûrs $(15 \mu)$ et atteint 25-30 $\mu$ au contact de la poche du cirre. Le canal déférent n'est entouré d'aucun manchon cellulaire. Il n'existe pas de vésicule séminale externe.

c) Poche du cirre (fig. 23). Ovalaire dans les anneaux jeunes, elle devient piriforme dans les anneaux mûrs. La poche du cirre est le p'us souvent perpendiculaire au bord du prog'ottis, ou très légèrement ob.ique, l'extrémité proxima!e étant alors la plus antérieure.

Les canaux osmorégulateurs passent dans la zone comprise entre la vésicule séminale interne et les longues soies tapissant l'intérieur de la poche constituée par le cirre invaginé. La poche du cirre mesure, dans les anneaux mûrs, $200 \mu$ de long (extrêmes : $140-230 \mu$ ) sur $100 \mu$ de large $(85-120 \mu)$, de sorte qu'el'e occupe une grande partie de la largeur du proglottis $(2 / 5$ à $1 / 3)$.

La paroi mince, dont l'épaisseur est comprise entre 1,5 et $3,5 \mu$, est constituée de fibres musculaires longitudinales. Le canal déférent donne naissance à une vésicule séminale interne $(40 \times 50 \mu)$. Fait suite à celle-ci un canal de 12 à $15 \mu$ de diamètre et de 35 à $50 \mu$ de long, dont la lumière est garnie de longues soies. Le canal est entouré de fibres musculaires disposées radialement par rapport à lui. Après avoir esquissé une boucle, il s'élargit pour donner la portion du cirre susceptible d'être évaginée. Cette région est tapissée dans sa partie proximale, sur une longueur d'environ $50 \mu$, de longues soies de 20 à $25 \mu$ de long. Dans sa partie distale, la paroi est inerme, principalement 
constituée dans son épaisseur $(5 \mu)$ de fibres musculaires circulaires. Cette paroi est plissée lorsque la poche du cirre n'est plus gonflée.

Dans cette espèce, l'autofécondation entre éléments mâles et femelles de chaque proglottis est obligatoire.

Plusieurs observations le long du strobile nous permettent de le constater. Sur aucun des Cestodes étudiés, nous n'avons trouvé de cirre évaginé. Vers le $165^{\circ}$ proglottis, avant le développement de l'ovaire, la poche du cirre est fortement dilatée. Son extrémité s'abouche à celle du vagin qui se dilate à son tour vers le $170^{\circ}$ proglottis. Cette disposition se retrouve du $170^{\circ}$ au $185^{\circ}$ proglottis. Le sperme ne s'accumule dans le vagin que vers le $177^{\circ}$ proglottis, alors que l'atrium génital ne communique pas encore avec l'extérieur. L'activité testiculaire s'effectue donc entre le $150^{\circ}$ et le $177^{\circ}$ proglottis où elle atteint son maximum.

\section{Appareil reproducteur femelle.}

a) Vagin. D'un diamètre variant de 35 à $45 \mu$, il débouche dans l'atrium génital, en arrière de la poche du cirre. Le vagin présente trois aspects différents se'on la position du proglottis auquel il appartient, sur le strobile (fig. $23 a, b, c$ ). Entre le $170^{\circ}$ et le $185^{\circ}$ proglottis, le vagin est fortement dilaté $(80 \mu$ de long $\times 45 \mu$ de large). La lumière du canal vaginal, à ce stade, a un diamètre de $35 \mu$. Elle est tapissée de très fines soies. Le sperme s'y accumu'e vers le $177^{\circ}$ anneau. La paroi est constituée d'une zone hyaline fibreuse, renforcée à l'extérieur par une couche de fibres musculaires circulaires (fig. $23 a$ ).

Dans les proglottis suivants, le vagin se contracte. Sa lumière devient étroite et les soies qui la garnissent se distinguent mieux. L'épaisseur de sa paroi augmente (dimensions : 70-100 $\times 35-40 \mu$ ). Elle est due principa'ement à la zone hyaline, fibreuse. Cette contraction du vagin s'accompagne d'une augmentation du volume du canal séminal jouant alors le rôle d'un réceptac'e séminal (fig. $23 \mathrm{~b}$ ).

Le vagin s'évagine à l'extérieur du prog'ottis, libérant à l'extérieur les soies qui tapissaient la lumière, et qui mesurent 5 à $6 \mu$ de long. Ce bourrelet est constitué dans sa plus grande part par la paroi la plus postérieure du vagin (fig. $23 \mathrm{c}$ ).

Le canal séminal s'élargit par endroits, atteignant 20 à $25 \mu$ de diamètre. Il décrit une courbe dans la région antérieure de l'ovaire et s'arrête au niveau de la glande vitellogène.

b) Ovaire. Ventral, constitué de deux masses plus ou moins lobées, l'ovaire se situe au centre du proglottis, entouré par les testicuies. Il est légèrement décalé du côté antiporal et la masse ovarienne antiporale est plus volumineuse que la porale.

c) Glande vitellogène. Compacte, de forme variable, généralement ovalaire, elle est ventrale. Dans les anneaux mûrs, elle mesure en moyenne 80-85 $\mu \times 55 \mu$. Contrairement à l'ovaire, son involution est lente et on la distingue encore dans les proglottis en début de gravidité. La g'ande de Mehlis n'est pas visible.

d) Utérus. A maturité, il se résout en capsules ovifères (fig. 22). Chaque proglottis gravide comprend de 9 à 12 capsules de 100 à $130 \mu$ de diamètre. Chacune d'elles ren- 
ferme de 11 à 15 œufs. D'abord étroitement accolées, les capsules ovifères se séparent les unes des autres dans les proglottis gravides qui augmentent de volume.

Les mensurations des œufs sont les suivantes :

- coque externe de l'œuf: 30-38 $\times 40-45 \mu$;

- oncosphère : $15 \times 17 \mu$. Les trois paires de crochets ne sont pas encore visibles dans les proglottis gravides que nous possédons.

En résumé, les caractères essentiels de ce Cestode sont les suivants : Cestode de 15 à $20 \mathrm{~mm}$ de long, 250 proglottis environ. Largeur du scolex : 250-285 $\mu$, rostre avec double couronne de crochets, de 340 à 424 crochets, longs de 13 à $14 \mu$, quatre ventouses armées de $80 \times$ $70 \mu$ de diamètre. Deux paires de canaux osmorégulateurs. Largeur des proglottis :

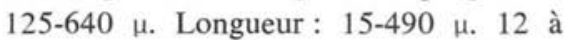
18 testicules. Poche du cirre : $140-230 \mu$ de large. Vagin : 35 à $45 \mu$ de long. 8 à 12 capsules ovifères par proglottis, contenant chacun 11 à 15 œufs de 30 $38 \times 40-45 \mu$ de diamètre.

\section{2) DISCUSSION :}

Trente-sept espèces appartenant au

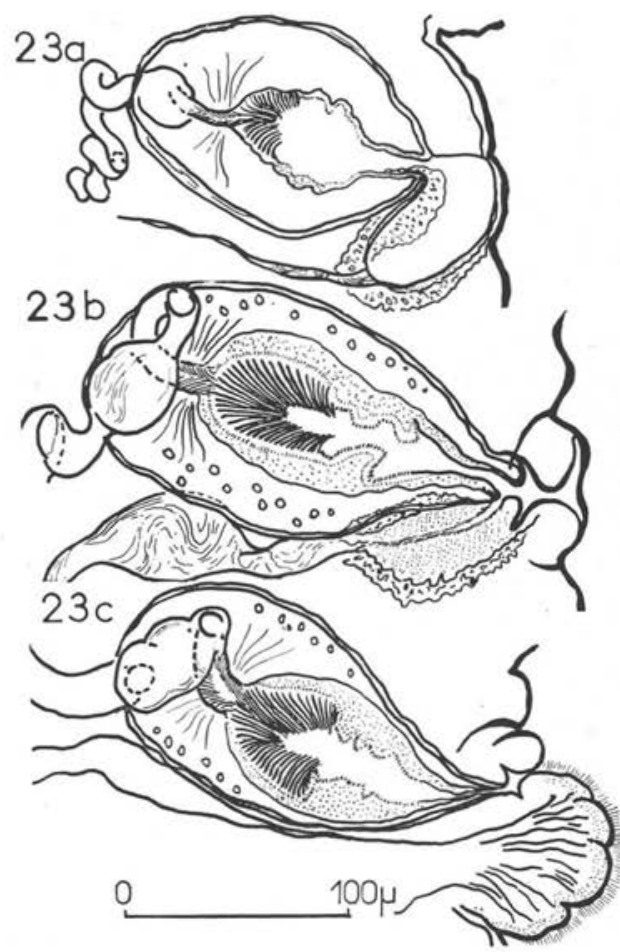

FIG. 23. - Raillietina (R.) ortleppi n. sp. Evolution de la poche du cirre et du vagin le long du strobile: a) $177^{\circ}$ proglottis ; b) $190^{\circ}$ proglottis ; c) $200^{\circ}$ proglottis. sous-genre Raillietina sont actuellement connues chez les Columbiformes. Les caractères spécifiques du Cestode étudié, énumérés ci-dessus, nous ont permis de limiter la comparaison à quatre espèces dont le rostellum possède plus de 300 crochets mesurant au moins $10 \mu$ :

- Raillietina (R.) johri Ortiepp 1938, chez Columba livia domestica Gmelin (Lucknow, Inde), diffère de notre espèce principalement par le nombre de ses testicules (6 à 7) et la faible longueur de la poche du cirre : $101 \mu$.

- R. (R.) clerci (Fuhrmann 1909), chez Columba sp. (Oural), possède une couronne de 400 crochets de $10 \mu$ de long. Cependant, les proportions de ce ver ne correspondent pas à nos mesures. $R$. (R.) clerci atteint $150 \mathrm{~mm}$ de long. Les exemplaires les plus longs que nous possédons ont une longueur de 15 à $20 \mathrm{~mm}$. Le nombre des testicules (20) est supérieur à celui relevé sur nos différents échantillons. 
- R. (R.) delalandei Ortlepp 1938, chez Vinago delalandei Bonaparte (Afrique du Sud), porte sur son rostellum 600 à 700 crochets. La poche du cirre est relativement petite puisqu'elle mesure 110 à $120 \mu$. Nous ne pouvons donc identifier nos échantillons à cette espèce.

- R. (R.) vinagoi Ortlepp 1938, chez Vinago delalandei Bonaparte (Transval et Natal), présente la plus grande analogie avec l'espèce décrite, par le nombre de crochets : 350 , et la longueur de la poche du cirre : $165-180 \mu$. Mais le nombre de testicules est supérieur (18-30) à celui de notre espèce (12-18) ; divers détails morphologiques sont différents, tels que la largeur du scolex, qui est ici double, et la position de l'atrium génital sur le bord du proglottis. En conséquence, ne pouvant identifier notre espèce à aucune de celles décrites précédemment, nous la considérons comme nouvelle et la nommons $R$. (R.) ortleppi n. sp., en hommage à R.-J. Ortlepp qui a contribué à la connaissance des Raillietina de Columbiformes.

\section{Résumé}

Deux Raillietina (Raillietina) ont été récoltés chez un Turacus sp. (Cuculiforme). Le premier présente les caractères morphologiques et anatomiques de $R$. (R.) khalili décrit par Hilmy en 1936. Le second : $R$. ( $R$.) douceti n. sp., est, parmi les cinq espèces de $R$. (R.) déjà décrites chez les Cuculiformes, proche par le nombre et la longueur des crochets du rostellum de $R$. (R.) calcaria (Fuhrmann 1909) et de $R$. (R.) macrocirrosa (Fuhrmann 1909). Il se distingue de ces deux Cestodes principalement par l'anatomie de l'atrium génital, celle de la poche du cirre, et par le nombre de testicules.

Raillietina (R.) ortleppi n. sp., parasite d'un Vinago waahli (Columbiforme), possède le même nombre de crochets sur le rostellum et une poche du cirre de mêmes dimensions que $R$. (R.) vinagoi Ortlepp 1938. Il en diffère cependant par un nombre de testicules moindre et un scolex deux fois plus petit.

\section{Bibliographie}

BAER (J.-G.), 1925. - Sur quelques Cestodes du Congo belge. Rev. Suisse Zool., XXXII, 239-251.

- 1933. - Contribution à l'étude de la faune helminthologique africaine. Ibid., XL, 31-84.

Fuhrmann (O.), 1909. - Neue Davaineiden. Central. Bakt. Parasitenk., I. Abt., Orig., IL, 94-124.

- 1920. - Considérations générales sur les Davainea. Festschrift f. Zschokke., Bâle, 1920, 19 p.

Hilmy (J. G.), 1936. - Parasites from Liberia and French Guinea. Part. III. Cestodes from Liberia. Publ. Egyptian Univ. Fac. Med., IX, 1-72.

Meggitt (F. G.), 1924. - The Tapeworms of the Rangoon pigeon. Parasit., XVI, 303-312.

ORTLePP (R. G.), 1938. - South African Helminths. Part. IV. Cestodes from Columbiformes. Onderstepoort. J. Vet. Sc. Anim. Ind., II, 51-61.

[Muséum National d'Histoire Naturelle, Paris, Laboratoire de Zoologie (Vers.)] 